\title{
The role of hypoglycemia in the burden of living with diabetes among adults with diabetes and family members: results from the DAWN2 study in The Netherlands
}

Giesje Nefs ${ }^{1,2,3^{*}}$ and François Pouwer ${ }^{1,4}$

\begin{abstract}
Background: To examine the relation between self-reported hypoglycemic events, worries about these episodes, and the burden of diabetes in adults with diabetes and family members from The Netherlands.

Methods: As part of the second multinational Diabetes Attitudes, Wishes and Needs (DAWN2) study, 412 Dutch adults with type 1 or type 2 diabetes and 86 family members completed questions about the burden of living with diabetes, the frequency of hypoglycemia, worries about these events, and several demographic and clinical factors. Analyses included hierarchical logistic regression.

Results: In total, $41 \%$ of people with diabetes and $56 \%$ of family members considered diabetes at least somewhat of a burden. In people with diabetes, diabetes burden was independently associated with self-reported current insulin use (fully adjusted $\mathrm{OR}=2.75,95 \% \mathrm{Cl} 1.49-5.10$ ), self-reported frequent non-severe hypoglycemia in the past year (OR= $2.45,1.25-4.83)$, self-reported severe hypoglycemia in the past year $(\mathrm{OR}=1.91,1.02-3.58)$, and being very worried about hypoglycemia at least occasionally $(\mathrm{OR}=3.64,2.18-6.10)$. For family members, the odds of experiencing living with diabetes as a burden was increased only for participants who were at least occasionally very worried about hypoglycemia (adjusted OR=5.07, 1.12-23.00).

Conclusions: Approximately half of adults with diabetes and adult family members experienced at least some diabetes burden. In both groups, diabetes burden appeared to be associated with being very worried about hypoglycemia at least occasionally. If these results are replicated, new intervention studies could test new ways of decreasing the traumatic consequences of previous or anticipated hypoglycemic events for people with diabetes and family members.
\end{abstract}

Keywords: Hypoglycemia, Worries, Burden, Family members

\section{Background}

Despite ongoing improvements in technology and care, living with diabetes remains a challenge for many people. The second Diabetes Attitudes, Wishes and Needs (DAWN2) study among 8596 adults with diabetes across 17 countries found that $12 \%$ of participants

\footnotetext{
* Correspondence: g.m.nefs@tilburguniversity.edu

${ }^{1}$ CoRPS - Center of Research on Psychological and Somatic disorders, Department of Medical and Clinical Psychology, Tilburg University, PO BOX 90153, 5000 LE Tilburg, the Netherlands

${ }^{2}$ Diabeter, National treatment and research center for children, adolescents and young adults with type 1 diabetes, Rotterdam, The Netherlands Full list of author information is available at the end of the article
}

reported poor or very poor overall quality of life, $14 \%$ had likely depression and $45 \%$ showed high diabetesspecific distress [1]. In addition, diabetes had a negative impact on all examined aspects of life, including physical health and relationships [1]. Diabetes and its treatment not only affect the person living with this condition, but may also have an impact on family members [2]. The DAWN2 study surveyed 2057 adult family members and found that over one third perceived a considerable burden of diabetes on the family [3]. People with diabetes and their family members may experience a range of worries about risks connected with diabetes, with 
hypoglycemia featuring as one of the key themes (Hermanns et al., submitted; [4]).

Hypoglycemia remains the greatest barrier to achieving and maintaining tight glycemic control in people with type 1 diabetes and people with type 2 diabetes on insulin therapy or sulfonylurea treatment [5]. The prevalence of severe hypoglycemia (requiring assistance from another person for recovery [6]) varies according to diabetes type and treatment duration, ranging from $7 \%$ in people with type 2 diabetes using sulfonylurea or insulin for less than 2 years to $46 \%$ in those with longstanding type 1 diabetes (>15 years) [7]. Non-severe hypoglycemia (events manageable by the person with diabetes) is also an important problem as symptoms can be unpleasant and aversive, and may interfere with following treatment recommendations $[8,9]$.

Findings of the international DAWN2 study among adults with diabetes and family members of people with diabetes suggest psychological outcomes are worse when hypoglycemia is more frequent $[10,11]$. However, substantial between-country variation in outcomes and associations in these studies underlines the need for country-specific analyses $[10,11]$. Therefore, the present study used data from the Dutch sample of the DAWN2 study to examine the relation between the frequency of hypoglycemia, worries about these events, and the burden of diabetes in adults with diabetes and family members from The Netherlands.

\section{Methods}

\section{Procedure and participants}

The second Diabetes Attitudes Wishes and Needs (DAWN2) study used a cross-sectional questionnaire survey in 17 countries across four continents, to examine the attitudes, wishes and needs of people with diabetes, family members and healthcare professionals [12]. Design and procedures for the Dutch sub study were in line with the international study protocol [12]. For the sample of people with diabetes, inclusion criteria were age $\geq 18$ years, a diagnosis of (non-gestational) diabetes by a healthcare professional at least 12 months ago, and disclosure of current diabetes treatment. For the sample of family members, inclusion criteria were age $\geq 18$ years, not having diabetes yourself, living in the same household with an adult with (non-gestational) diabetes diagnosed at least 12 months ago, and being involved in their diabetes care. Potential participants were initially identified via existing panels and databases. Individuals were then contacted via email or by phone. Interviews were conducted online (self-administered), by phone, or face-to-face (for a small number). The study was conducted in accordance with ethical requirements and all participants provided informed consent.
The Dutch subsamples included 502 adults with diabetes and 120 family members. For the present analyses, we excluded people taking injectables other than insulin ("non-insulin injectables", e.g. GLP-1 agonists) as there were relatively few of them $(n=15)$ and their data might cloud the results. We also excluded people not meeting the definition of type 1 and type 2 diabetes as employed in DAWN2 [1]. Type 1 diabetes was defined as selfreported (a) diagnosis before the age of 30, (b) prescription of insulin when first diagnosed, and (c) current insulin use. Type 2 diabetes was defined as self-reported (a) diagnosis on or after the age of 30, and (b) no prescription of insulin when first diagnosed. This left 412 people with diabetes and 86 family members.

\section{Measurements \\ Diabetes burden}

As a proxy of diabetes burden in people with diabetes, we used an item from the Problem Areas in Diabetes questionnaire [13]. Participants were asked to indicate on a five-point Likert scale ranging from "not a problem" to "serious problem" whether "Feeling that diabetes is taking up too much of your mental and physical energy every day" was currently a problem for them. Diabetes was considered a burden if participants reported a minor, moderate, somewhat serious, or serious problem. We preferred the pooling of people with minor and major concerns over the pooling of people with minor and no concerns, as this last approach would ignore a group who do report a problem (albeit not of major proportions). Family members were asked to indicate on a five-point Likert scale ranging from "no burden" to "very large burden" how much of a burden it was for them to help manage the diabetes of the person they lived with. Diabetes was considered a burden if participants reported a slight, moderate, large or very large burden.

\section{Hypoglycemic events}

People with diabetes and family members were asked to report the average number of times the person with diabetes has had symptoms of hypoglycemia, which they could treat themselves during the past 12 months. Based on clinical experience and the distribution of replies, frequency of non-severe hypoglycemia was categorized as "none" (no symptoms in past 12 months), "occasional" (once a month, less often than once a month) and "frequent" (at least once a day, at least once a week, several times a month). In addition, both groups were asked to report approximately how many times during the past 12 months the person with diabetes has had severely low blood sugar that he/she was unable to treat himself/ herself and needed help from someone to restore blood sugar levels. Severe hypoglycemia during the past 
12 months was categorized as "no" versus "one or more events".

\section{Worries about hypoglycemia}

People with diabetes and family members were asked to rate on a four-point Likert scale to what extent they agreed with the statement "I am very worried about the risk of [him/her having] hypoglycemic (low blood sugar) events". When they mainly disagreed, mainly agreed and fully agreed with the statement, this was taken to indicate the presence of at least some worries about hypoglycemia.

\section{Demographic and clinical covariates}

For both people with diabetes and family members, variables included gender, age, educational level (with a high level defined as having completed higher vocational education or university), working full or part-time (no/yes), and whether the person with diabetes was using insulin to manage diabetes (no/yes). For people with diabetes, additional information included whether they were currently living with somebody (no/yes), diabetes duration, and the presence of one or more (potentially diabetes- related) co-morbid conditions (stroke, foot ulcer, foot/ leg amputation, kidney disease, eye damage, nerve damage, heart disease).

\section{Statistical analyses}

Two separate hierarchical (blockwise) logistic regression analyses were used (one for people with diabetes and one for family members) to examine the association of hypoglycemic events and worries about hypoglycemia with diabetes burden. For family members, step 1 included gender, age, educational level, employment, insulin use, non-severe hypoglycemia and severe hypoglycemia. In step 2, worries about hypoglycemia were added. For people with diabetes, living situation, diabetes duration and co-morbidities were also included in step 1. All analyses were performed using SPSS Version 22 (IBM SPSS Statistics, New York). A $p$-value $<0.05$ indicated statistical significance.

\section{Results}

Sample characteristics

Descriptive characteristics of the samples of adults with diabetes and family members are displayed in Table 1.

Table 1 Descriptive characteristics of the sample of adults with diabetes and adult family members

\begin{tabular}{|c|c|c|c|c|}
\hline & Missing values & Adults with diabetes $(n=412)$ & Missing values & Family members $(n=86)$ \\
\hline Female gender & 0 & $48 \%(197)$ & 0 & $83 \%(71)$ \\
\hline Age, years & 0 & $60 \pm 11(62,54-67)$ & 0 & $54 \pm 13(56,50-62)$ \\
\hline High educational level & 17 & $26 \%(102)$ & 3 & $35 \%(29)$ \\
\hline Living alone & 0 & $27 \%(113)$ & & NA \\
\hline Working full- or parttime & 2 & $27 \%(112)$ & 0 & $38 \%(33)$ \\
\hline Diabetes type and treatment & 0 & & 0 & \\
\hline Type 2 diabetes, no medication & & $22 \%(91)$ & & $15 \%(13)$ \\
\hline Type 2 diabetes, oral medication only & & $36 \%(150)$ & & $51 \%(44)$ \\
\hline Type 2 diabetes, insulin & & $22 \%(91)$ & & $26 \%(22)$ \\
\hline Type 1 diabetes & & $19 \%(80)$ & & $8 \%(7)$ \\
\hline Diabetes duration, years & 0 & $13 \pm 12(9,4-17)$ & & NA \\
\hline Co-morbidities & 0 & & & NA \\
\hline No & & $45 \%(184)$ & & \\
\hline One & & $30 \%(125)$ & & \\
\hline Two or more & & $25 \%(103)$ & & \\
\hline Non-severe hypoglycemia in past year & 3 & & 5 & \\
\hline None & & $33 \%(134)$ & & $40 \%(32)$ \\
\hline Occasional & & $32 \%(132)$ & & $36 \%(29)$ \\
\hline Frequent & & $35 \%(143)$ & & $25 \%(20)$ \\
\hline Severe hypoglycemia in past year ( $\geq 1$ event) & 1 & $20 \%(83)$ & 22 & $30 \%(19)$ \\
\hline Worries about hypoglycemia & 19 & $57 \%(225)$ & 7 & $61 \%(48)$ \\
\hline Diabetes burden & 0 & $41 \%(170)$ & 0 & $56 \%(48)$ \\
\hline
\end{tabular}

NA not applicable/available

Values are $\%(n)$ or mean \pm SD (median, interquartile range) 
Compared to people with diabetes, family members appeared to be more likely to be female, to have a high educational level, and to be employed, and were somewhat younger. In the sample of people with diabetes, participants with type 1 diabetes were somewhat overrepresented compared to national estimates [14]. People with diabetes were somewhat more likely to report frequent non-severe hypoglycemia, while family members more commonly indicated that the person with diabetes they lived with had experienced severe hypoglycemia during the past 12 months. Approximately 60\% of people with diabetes and family members were at least occasionally very worried about hypoglycemia, while living with diabetes was a small to large burden for half of participants in both groups.

\section{Diabetes burden in people with diabetes}

When the frequency of non-severe and severe hypoglycemia were entered in step 1 along with the demographic and clinical covariates, this first model was statistically significant $\left(\chi^{2}(12)=72, p<0.001\right.$; Cox and Snell $R$ square $=0.17$; Nagelkerke $R$ square $=0.23$ ). As shown in Table 2, participants who used insulin $(p=$ 0.003), who experienced frequent non-severe hypoglycemia during the past 12 months (compared to people reporting none of these events; $p<0.001)$ and who had experienced a severe hypoglycemic event during the past 12 months $(p=0.02)$ had higher odds of considering diabetes at least somewhat of a burden, adjusted for the other variables in model 1. Entry of worries about hypoglycemia in step 2 led to a significant model improvement (change $\chi^{2}(1)=26, p<0.001$; Cox and Snell R square $=0.23$; Nagelkerke $R$ square $=0.31$ ) . In this fully adjusted model $\left(\chi^{2}(13)=98, p<0.001\right)$, participants who used insulin $(p=0.001)$, who experienced frequent non-severe hypoglycemia during the past 12 months (compared with people reporting none of these events; $p=0.009$ ), who had experienced a severe hypoglycemic event during the past 12 months ( $p=$ $0.04)$, and who were at least occasionally very worried about hypoglycemia $(p<0.001)$ had higher odds of considering diabetes at least somewhat of a burden.

\section{Diabetes burden in family members}

When the frequency of non-severe and severe hypoglycemia were entered in step 1 along with the demographic and clinical covariates, this first model was not statistically significant $\left(\chi^{2}(8)=8, p=0.42\right.$; Cox and Snell R square 0.13; Nagelkerke $R$ square 0.18). As shown in Table 2, none of the demographic and clinical variables in this step were independently associated with diabetes burden in family members. Entry of worries about hypoglycemia in step 2 led to a significant model improvement (change $\chi^{2}(1)=5, p=0.03$; Cox and Snell $R$ square $=0.20$; Nagelkerke $R$ square $=0.27$ ). However, this fully adjusted model was still not statistically

Table 2 The relation between demographic, clinical and psychological factors and the burden of diabetes

\begin{tabular}{|c|c|c|c|c|}
\hline & \multicolumn{2}{|c|}{ Adults with diabetes $(n=377)$} & \multicolumn{2}{|c|}{ Family members $(n=58)$} \\
\hline & Model 1 & Model 2 & Model 1 & Model 2 \\
\hline Female gender & $1.04(0.64-1.69)$ & $1.08(0.65-1.79)$ & $1.24(0.23-6.87)$ & $1.00(0.16-6.12)$ \\
\hline Age, years & $0.98(0.95-1.01)$ & $0.98(0.95-1.01)$ & $0.99(0.94-1.04)$ & $0.98(0.93-1.03)$ \\
\hline High educational level & $0.92(0.53-1.58)$ & $1.09(0.62-1.94)$ & $0.53(0.13-2.11)$ & $0.99(0.21-4.66)$ \\
\hline Living alone & $1.24(0.74-2.09)$ & $1.23(0.71-2.10)$ & NA & NA \\
\hline Working full- or parttime & $0.70(0.39-1.24)$ & $0.66(0.36-1.20)$ & $1.67(0.43-6.44)$ & $0.98(0.22-4.35)$ \\
\hline Diabetes duration, years & $0.99(0.97-1.01)$ & $0.99(0.96-1.01)$ & NA & NA \\
\hline Using insulin & $2.45(1.36-4.41)$ & $2.75(1.49-5.10)$ & $1.57(0.37-6.77)$ & $0.86(0.17-4.35)$ \\
\hline Co-morbidities & & & NA & NA \\
\hline No & Ref & Ref & & \\
\hline One & $1.36(0.79-2.35)$ & $1.36(0.77-2.38)$ & & \\
\hline Two or more & $1.00(0.54-1.86)$ & $1.04(0.55-1.98)$ & & \\
\hline \multicolumn{5}{|l|}{ Non-severe hypoglycemia in past year } \\
\hline None & Ref & Ref & Ref & Ref \\
\hline Occasional & $1.75(0.95-3.23)$ & $1.33(0.70-2.52)$ & $1.15(0.26-5.06)$ & $0.69(0.13-3.53)$ \\
\hline Frequent & $3.55(1.87-6.76)$ & $2.45(1.25-4.83)$ & $3.84(0.65-22.79)$ & $1.52(0.20-11.34)$ \\
\hline Severe hypoglycemia in past year ( $\geq 1$ event) & $2.00(1.09-3.66)$ & $1.91(1.02-3.58)$ & $1.51(0.34-6.78)$ & $2.28(0.44-11.87)$ \\
\hline Worries about hypoglycemia & & $3.64(2.18-6.10)$ & & $5.07(1.12-23.00)$ \\
\hline
\end{tabular}

NA not applicable/available

Hierarchical logistic regression analyses. Values are OR $(95 \% \mathrm{Cl})$; bold = statistically significant $(p<0.05)$ 
significant $\left(\chi^{2}(9)=13, p=0.16\right)$. Family members who were at least occasionally very worried about hypoglycemia $(p=0.04)$ had higher odds of considering diabetes at least somewhat of a burden, adjusted for the other variables in model 2 .

\section{Discussion}

This study based on the Dutch subsample of the international DAWN2 study found that almost half of adults with diabetes considered diabetes at least somewhat of a burden. A direct comparison with figures from other countries is difficult to make. While we based the definition of diabetes burden on one item focusing on the mental and physical costs of living with this condition, the cross-national benchmarking paper of the DAWN2 study considered several more global measures including the composite score of the Problem Areas in Diabetes 5 item version and the DAWN Impact of Diabetes Profile [1]. Countries ranked differently across these different indicators of diabetes burden, where no individual country or region appeared to be consistently better or worse than the others [1]. Overall, the Dutch sample appeared to be among the quarter of countries with the most optimal scores in this domain [1]. With respect to family members, we found that more than half of the Dutch participants indicated at least some burden in helping the person with diabetes they lived with managing their diabetes. The international DAWN2 papers employed a more strict definition of burden, finding 35\% of the total sample reporting a moderate to very large burden [3], with the Dutch subsample scoring slightly under the total mean [3].

In participants with diabetes, we found a positive association between self-reported insulin use and experienced diabetes burden, adjusted for the other variables. Previous studies have suggested that insulin therapy is viewed as the most burdensome treatment modality among people with type 2 diabetes [15]. While negative appraisals of insulin therapy are highest among those who do not have previous experience with insulin therapy, people who are currently using insulin or have prior experience with this treatment modality still experience substantial negative views about insulin [1517]. In the present study, the relation between selfreported insulin use and diabetes burden was independent of worries about hypoglycemia and self-reported occurrence of severe and non-severe hypoglycemic events in the previous year, suggesting other aspects of insulin treatment might play a role. For example, in the Diabetes MILES - Australia study half of participants with type 2 diabetes using insulin believed that taking insulin meant their diabetes had become worse and that insulin caused weight gain [16]. Furthermore, approximately $25 \%$ of adults with type 1 or insulin-treated type
2 diabetes experience injection-related anxiety [18, 19]. In addition, both of these groups frequently have to cope with actual or anticipated negative attention and judgment from others when self-injecting insulin by pen or pump [20-22].

With respect to hypoglycemia, previous studies have mostly focused on the psychological impact of severe events, finding a relation with greater diabetes-related distress and poorer general emotional well-being [23]. A large European study among people with type 1 diabetes and insulin-treated type 2 diabetes showed that nonsevere hypoglycemia may also have a substantial negative impact on energy and mood [24]. In the present study, people with diabetes also had increased odds of diabetes burden not only when they had experienced at least one self-reported severe hypoglycemic event, but also in case of self-reported frequent non-severe hypoglycemia during the past 12 months, adjusted for the other variables. The relation between proxy-reported hypoglycemic events and diabetes burden was not found in family members. This may be explained by the relatively small number of family members available for the regression analysis. Alternatively, it is possible that family members have not been aware of all hypoglycemic events that have occurred. From previous studies, it is known that people with diabetes and their family members often disagree about the frequency and nature of hypoglycemic episodes [25, 26].

In both people with diabetes and family members, more worries about hypoglycemia were associated with higher diabetes burden, adjusted for the other variables. While the recent occurrence of hypoglycemic events has been associated with higher worries about hypoglycemia $[23,27,28]$, the relation between worries about hypoglycemia and diabetes burden was independent of the occurrence of self- or proxy-reported severe and non-severe hypoglycemic events in the past year. Several studies from the pediatric diabetes literature suggest that, apart from the frequency of events, the types of experiences with hypoglycemia (e.g. with seizures or unconsciousness) may determine the level of experienced worries about future events [29-31]. A study among 90 adults with type 1 diabetes found that over 25\% met diagnostic criteria of hypoglycemia-specific posttraumatic stress and reported that perceived threat of death from hypoglycemia and fear of hypoglycemia were significantly related to these symptoms, while number of recent hypoglycemic episodes was not [32]. From the scientific literature on posttraumatic stress we know that it is also possible to experience symptoms triggered by anticipatory thoughts about future distressing events [33]. In family members, the extent to which they believe their relative with diabetes is able to cope with hypoglycemic episodes may also be an important 
determinant of the level of experienced worries about hypoglycemia. This hypothesis is supported by literature from the pediatric diabetes field, where trait anxiety levels and recent experiences with hypoglycemia predicted fear of hypoglycemia in adolescents with type 1 diabetes but beliefs about whether their child carried emergency glucose were associated with fear of hypoglycemia in parents [29].

The findings of this study should be considered in terms of its limitations and strengths. First, while crosscountry variations in outcomes necessitate the additional analysis for specific countries, the number of participating family members from the Netherlands was relatively low. This may have significantly underpowered the present analyses. Similarly, the number of participants in the present study does not allow stratifying the analyses for diabetes type. Also, unfortunately, the distribution of replies on the items about burden and worries and the increased number of analyses did not allow sensitivity analyses in which different definitions were used. Second, we had to rely on self- or proxy report about clinical variables such as diabetes type. Our decision to adhere to the previously used DAWN2 definitions of type 1 and type 2 diabetes based on age of diagnosis, insulin use shortly after diagnosis, and current insulin use [1] may have safeguarded against some of the biases inherent to self- or proxy report. However, we may have wrongfully excluded participants who would have qualified as type 1 or type 2 diabetes based on laboratory data, but diverge from the standard clinical picture on which the DAWN2 diabetes definitions were based. Furthermore, in this questionnaire-based study no objective clinical data (e.g. glycemic control, use of continuous glucose monitor) was available. Third, we used different definitions of diabetes burden in people with diabetes and family members, which makes a direct comparison of prevalence and correlates across these groups more difficult. Fourth, the main measures were based on single purpose-designed items rather than validated questionnaires. The one-item question from the PAID has not been officially validated as a proxy for diabetes burden, but was selected based on experience with the measure in clinical practice. Fifth, as one reviewer eloquently stated, "correlation does not mean causation". While we make some suggestions to guide intervention in the following paragraph, an experimental design is needed to establish whether manipulating worries about hypoglycemia actually lowers perceived disease burden. Finally, the results (especially for family members) should be interpreted with caution in light of several statistical considerations. Given the exploratory nature of the study, we did not adjust for multiple testing, with increased risk of type 1 errors. In addition, the number of observations is small relative to the number of predictors, increasing the risk of overfitting. The regression analysis for family members was run with a relatively high number of predictors to ensure optimal comparability with the analysis in people with diabetes, but it is prudent to repeat the analysis based on the larger international family members data set before strong conclusions are drawn. Also, even though we found a significant association between worries about hypoglycemia and diabetes burden in family members, it should be kept in mind that the test of the overall null for family members was not statistically significant. Strengths of the study include the focus on both people with diabetes and adult family members and the diverse demographic, (albeit self-reported) clinical and psychological correlates that were considered.

Most existing educational programs and behavioral interventions in the field of hypoglycemia to date have primarily focused on the avoidance of future events or improved awareness. These have generally found a reduction in the number of hypoglycemic episodes and an improvement in awareness, while improvements in worries about hypoglycemia were less consistent [34-37]. New technologies such as continuous glucose monitoring and sensor-augmented pump therapy have shown some promise in reducing hypoglycemia-related worries, but results are inconsistent and a more thorough evaluation of their potential with respect to patient-reported outcomes is warranted [38]. As memories of previous traumatic hypoglycemic events may be difficult to overcome, a focus on the prevention and management of hypoglycemia may not be enough to reduce fear of hypoglycemia for all adults with diabetes [38]. In this context, the anxiety and stress reducing properties of eye movement desensitization and reprocessing treatment should be explored further [33]. Interventions could also target worries about hypoglycemia in adult family members, as these may lead to suboptimal psychological outcomes of family members as well as suboptimal diabetes support $[4,11]$.

\section{Conclusions}

In conclusion, approximately half of adults with diabetes and adult family members experienced at least some diabetes burden. In both groups, being very worried about hypoglycemia at least occasionally appeared to be independently associated with diabetes burden. Other significant independent correlates with higher diabetes burden in people with diabetes included self-reported insulin use, self-reported frequent non-severe hypoglycemia and self-reported severe hypoglycemia. The merits of new technologies and educational / behavioral interventions with a primary focus on the prevention of hypoglycemic events and improved hypoglycemia awareness in reducing fear of hypoglycemia need to be explored further. 
However, these could be supplemented with intervention studies that test new treatments targeting the traumatic consequences of previous or future anticipated events and hypoglycemia (self)-efficacy. The role and worries of family members may also be considered. Given the disappointing reproducibility of scientific findings [39], replication of results is an important first step in this process.

\section{Abbreviations}

DAWN2 study: the second Diabetes Attitudes, Wishes and Needs study

\begin{abstract}
Acknowledgements
The authors thank all people with diabetes and family members who participated in DAWN2. The authors wish to acknowledge the contribution to the design and execution of the DAWN2 study of the DAWN2 Study Group (consisting of a lead national investigator from each country and members of the DAWN2 International Publication Planning Committee), Harris Interactive Inc., Bioscript Medical Ltd., the International Diabetes Federation and the numerous international and national experts and patient advocates.
\end{abstract}

\section{Funding}

The DAWN2 study is funded by Novo Nordisk A/S. In collaboration with national, regional and global partners, Novo Nordisk has assisted with the planning and designing of the DAWN2 study. Data collection was independently performed by Harris Interactive, who were funded by Novo Nordisk. Data analysis and manuscript preparation were performed by the authors.

\section{Availability of data and materials}

The data that support the findings of this study are available from Novo Nordisk but restrictions apply to the availability of these data, which were used under license for the current study, and so are not publicly available. Data are however available from the authors upon reasonable request and with permission of Novo Nordisk.

\section{Authors' contributions}

GN and FP contributed to the conception and design of the study, analysis and interpretation of the data; drafted the first version of the manuscript: critically revised the manuscript for important intellectual content; and approved the final version for publication. Both authors take responsibility for the contents of the article.

\section{Ethics approval and consent to participate}

The DAWN2 study was conducted in accordance with the relevant national/ regional/local guidelines relating to the conduct of non-interventional studies, using the guidelines of the International Chamber of Commerce / European Society for Opinion and Marketing Research, the Council of American Survey Research Organizations, and Good Pharmacoepidemiology Practices (UTN No: U1111-1123-7509; NCT01507116). All participants provided informed consent. The data were used with permission of Novo Nordisk.

\section{Consent for publication}

Not applicable

\section{Competing interests}

GN reports no conflict of interest. FP has received funding for travel and accommodation to attend DAWN2 International Publication Planning Committee meetings. His research group received a research grant from Novo Nordisk to conduct statistical analyses and describe results of the DAWN2 study and submit this work for publication.

\section{Publisher's Note}

Springer Nature remains neutral with regard to jurisdictional claims in published maps and institutional affiliations.

\section{Author details}

'CoRPS - Center of Research on Psychological and Somatic disorders, Department of Medical and Clinical Psychology, Tilburg University, PO BOX 90153, 5000 LE Tilburg, the Netherlands. ${ }^{2}$ Diabeter, National treatment and research center for children, adolescents and young adults with type 1 diabetes, Rotterdam, The Netherlands. ${ }^{3}$ Radboud university medical center, Radboud Institute for Health Sciences, Department of Medical Psychology, Nijmegen, The Netherlands. ${ }^{4}$ Department of Psychology, University of Southern Denmark, Odense, Denmark.

Received: 19 January 2017 Accepted: 10 January 2018

Published online: 18 January 2018

\section{References}

1. Nicolucci A, Kovacs Burns K, Holt Rl, Comaschi M, Hermanns N, Ishii H, Kokoszka A, Pouwer F, Skovlund SE, Stuckey H, et al. Diabetes attitudes, wishes and needs second study (DAWN2): cross-national benchmarking of diabetes-related psychosocial outcomes for people with diabetes. Diabet Med. 2013;30(7):767-77.

2. Rintala TM, Jaatinen P, Paavilainen E, Astedt-Kurki P. Interrelation between adult persons with diabetes and their family: a systematic review of the literature. J Fam Nurs. 2013;19(1):3-28.

3. Kovacs Burns K, Nicolucci A, Holt Rl, Willaing I, Hermanns N, Kalra S, Wens J, Pouwer F, Skovlund SE, Peyrot M. Diabetes attitudes, wishes and needs second study (DAWN2): cross-national benchmarking indicators for family members living with people with diabetes. Diabet Med. 2013;30(7):778-88.

4. Nefs G, Pouwer F, Holt RI, Skovlund S, Hermanns N, Nicolucci A, Peyrot M. Correlates and outcomes of worries about hypoglycemia in family members of adults with diabetes: the second diabetes attitudes, wishes and needs (DAWN2) study. J Psychosom Res. 2016;89:69-77.

5. Frier BM. How hypoglycaemia can affect the life of a person with diabetes. Diabetes Metab Res Rev. 2008;24(2):87-92.

6. Seaquist ER, Anderson J, Childs B, Cryer P, Dagogo-Jack S, Fish L, Heller SR, Rodriguez H, Rosenzweig J, Vigersky R. Hypoglycemia and diabetes: a report of a workgroup of the American Diabetes Association and the Endocrine Society. Diabetes Care. 2013;36(5):1384-95.

7. UK Hypoglycaemia Study Group. Risk of hypoglycaemia in types 1 and 2 diabetes: effects of treatment modalities and their duration. Diabetologia. 2007;50(6):1140-7.

8. McAulay V, Deary IJ, Frier BM. Symptoms of hypoglycaemia in people with diabetes. Diabet Med. 2001;18(9):690-705.

9. Alvarez Guisasola F, Tofe Povedano S, Krishnarajah G, Lyu R, Mavros P, Yin D. Hypoglycaemic symptoms, treatment satisfaction, adherence and their associations with glycaemic goal in patients with type 2 diabetes mellitus: findings from the real-life effectiveness and care patterns of diabetes management (RECAP-DM) study. Diabetes Obes Metab. 2008;10(Suppl 1): 25-32.

10. Nicolucci A, Kovacs Burns K, Holt RI, Lucisano G, Skovlund SE, Kokoszka A, Massi Benedetti M, Peyrot M. Correlates of psychological outcomes in people with diabetes: results from the second diabetes attitudes, wishes and needs (DAWN2) study. Diabet Med. 2016;33(9):1194-203.

11. Kovacs Burns K, Holt Rl, Nicolucci A, Lucisano G, Skovlund SE, Comaschi M, Vallis M, Peyrot M. Correlates of psychological outcomes among family members of people with diabetes in the second diabetes attitudes, wishes and needs (DAWN2) study. Diabet Med. 2016;33(9):1184-93.

12. Peyrot M, Burns KK, Davies M, Forbes A, Hermanns N, Holt R, Kalra S, Nicolucci A, Pouwer F, Wens J, et al. Diabetes attitudes wishes and needs 2 (DAWN2): a multinational, multi-stakeholder study of psychosocial issues in diabetes and person-centred diabetes care. Diabetes Res Clin Pract. 2013; 99(2):174-84.

13. Snoek FJ, Pouwer F, Welch GW, Polonsky WH. Diabetes-related emotional distress in Dutch and U.S. diabetic patients: cross-cultural validity of the problem areas in diabetes scale. Diabetes Care. 2000;23(9):1305-9.

14. Diabetes in cijfers [https://www.diabetesfonds.n//over-diabetes/diabetes-inhet-algemeen/diabetes-in-cijfers].

15. Vijan S, Hayward RA, Ronis DL, Hofer TP. Brief report: the burden of diabetes therapy: implications for the design of effective patient-centered treatment regimens. J Gen Intern Med. 2005;20(5):479-82.

16. Holmes-Truscott E, Skinner TC, Pouwer F, Speight J. Negative appraisals of insulin therapy are common among adults with type 2 diabetes using 
insulin: results from diabetes MILES - Australia cross-sectional survey. Diabet Med. 2015;32(10):1297-303.

17. Holmes-Truscott E, Skinner TC, Pouwer F, Speight J. Explaining psychological insulin resistance in adults with non-insulin-treated type 2 diabetes: the roles of diabetes distress and current medication concerns. Results from diabetes MILES-Australia. Prim Care Diabetes. 2016;10(1):75-82.

18. Zambanini A, Newson RB, Maisey M, Feher MD. Injection related anxiety in insulin-treated diabetes. Diabetes Res Clin Pract. 1999;46(3):239-46.

19. Mollema ED, Snoek FJ, Ader HJ, Heine RJ, van der Ploeg HM. Insulintreated diabetes patients with fear of self-injecting or fear of selftesting: psychological comorbidity and general well-being. J Psychosom Res. 2001;51(5):665-72.

20. Browne JL, Ventura A, Mosely K, Speight J. 'I call it the blame and shame disease': a qualitative study about perceptions of social stigma surrounding type 2 diabetes. BMJ Open. 2013;3(11):e003384.

21. Browne JL, Ventura A, Mosely K, Speight J. I'm not a druggie, I'm just a diabetic': a qualitative study of stigma from the perspective of adults with type 1 diabetes. BMJ Open. 2014;4(7):e005625.

22. Browne JL, Ventura AD, Mosely K, Speight J. Measuring the stigma surrounding type 2 diabetes: development and validation of the type 2 diabetes stigma assessment scale (DSAS-2). Diabetes Care. 2016; 39(12):2141-8.

23. Hendrieckx C, Halliday JA, Bowden JP, Colman PG, Cohen N, Jenkins A, Speight J. Severe hypoglycaemia and its association with psychological well-being in Australian adults with type 1 diabetes attending specialist tertiary clinics. Diabetes Res Clin Pract. 2014;103(3):430-6.

24. Geelhoed-Duijvestijn P, Pedersen-Bjergaard U, Weitgasser R, Lahtela J, Markert Jensen M, Ostenson CG. Effects of patient-reported non-severe hypoglycemia on healthcare resource use, work-time loss, and wellbeing in insulin-treated patients with diabetes in severe European countries. J Med Econ. 2013;16(12):1453-61.

25. Heller S, Chapman J, McCloud J, Ward J. Unreliability of reports of hypoglycaemia by diabetic patients. BMJ. 1995;310(6977):440.

26. Jorgensen HV, Pedersen-Bjergaard U, Rasmussen AK, Borch-Johnsen K. The impact of severe hypoglycemia and impaired awareness of hypoglycemia on relatives of patients with type 1 diabetes. Diabetes Care. 2003;26(4): $1106-9$.

27. Nefs G, Bevelander S, Hendrieckx C, Bot M, Ruige J, Speight J, Pouwer F. Fear of hypoglycaemia in adults with type 1 diabetes: results from diabetes MILES - The Netherlands. Diabet Med. 2015;32(10):1289-96.

28. Sakane N, Kotani K, Tsuzaki K, Nishi M, Takahashi K, Murata T, Yamada K, Okazaki K, Yanagisawa K, Yamada K, et al. Fear of hypoglycemia and its determinants in insulin-treated patients with type 2 diabetes mellitus. J Diabetes Investigation. 2015;6(5):567-70.

29. Gonder-Frederick LA, Fisher CD, Ritterband LM, Cox DJ, Hou L, DasGupta AA, Clarke WL. Predictors of fear of hypoglycemia in adolescents with type 1 diabetes and their parents. Pediatr Diabetes. 2006;7(4):215-22.

30. Marrero DG, Guare JC, Vandagriff JL, Fineberg NS. Fear of hypoglycemia in the parents of children and adolescents with diabetes: maladaptive or healthy response? Diabetes Educ. 1997;23(3):281-6.

31. Clarke WL, Gonder-Frederick A, Snyder AL, Cox DJ. Maternal fear of hypoglycemia in their children with insulin dependent diabetes mellitus. $J$ Pediatr Endocrinol Metab. 1998;11(Suppl 1):189-94.

32. Myers VH, Boyer BA, Herbert JD, Barakat LP, Scheiner G. Fear of hypoglycemia and self reported posttraumatic stress in adults with type 1 diabetes treated by intensive regimens. J Clin Psychol Med Settings. 2007; 14:11-21.

33. Logie RDJ, De Jongh A. The "flashforward procedure": confronting the catastrophe. J EMDR Pract Res. 2014;8(1):25-32

34. Cox DJ, Gonder-Frederick L, Ritterband L, Patel K, Schachinger H, FehmWolfsdorf G, Hermanns N, Snoek F, Zrebiec J, Polonsky W, et al. Blood glucose awareness training: what is it, where is it, and where is it going? Diabetes Spectr. 2006;19(1):43-9.

35. de Zoysa N, Rogers H, Stadler M, Gianfrancesco C, Beveridge S, Britneff E, Choudhary P, Elliott J, Heller S, Amiel SA. A psychoeducational program to restore hypoglycemia awareness: the DAFNE-HART pilot study. Diabetes Care. 2014;37(3):863-6.

36. Rondags SM, de Wit M, Twisk JW, Snoek FJ. Effectiveness of HypoAware, a brief partly web-based Psychoeducational intervention for adults with type 1 and insulin-treated type 2 diabetes and problematic hypoglycemia: a cluster randomized controlled trial. Diabetes Care. 2016;39(12):2190-6.
37. Little SA, Leelarathna L, Walkinshaw E, Tan HK, Chapple O, Lubina-Solomon A, Chadwick TJ, Barendse S, Stocken DD, Brennand C, et al. Recovery of hypoglycemia awareness in long-standing type 1 diabetes: a multicenter 2 $\times 2$ factorial randomized controlled trial comparing insulin pump with multiple daily injections and continuous with conventional glucose selfmonitoring (HypoCOMPaSS). Diabetes Care. 2014;37(8):2114-22.

38. Martyn-Nemeth P, Schwarz Farabi S, Mihailescu D, Nemeth J, Quinn L. Fear of hypoglycemia in adults with type 1 diabetes: impact of therapeutic advances and strategies for prevention - a review. J Diabetes Complicat. 2016;30(1):167-77.

39. Open Science Collaboration, Psychology. Estimating the reproducibility of psychological science. Science. 2015;349(6251):aac4716.

\section{Submit your next manuscript to BioMed Central and we will help you at every step:}

- We accept pre-submission inquiries

- Our selector tool helps you to find the most relevant journal

- We provide round the clock customer support

- Convenient online submission

- Thorough peer review

- Inclusion in PubMed and all major indexing services

- Maximum visibility for your research

Submit your manuscript at www.biomedcentral.com/submit
Biomed Central 